Received 23 April; accepted 18 July 1979

1. Luck, W., Klier, M. \& Wesslau, H. Ber. Bunseg. Phys. Chem. 67, 75-83 (1963)

2. Hiltner, P. A. \& Krieger, I. M. J. phys. Chem. 73, 2386-2391 (1969).

3. Williams, R. \& Crandall, R. S. Phys. Lett. 48A, 225-226 (1974).

4. Kose, A., Ozaki, M., Takano, K., Kobayashi, Y. \& Hachisu, S. J. Coll. Int. Sci. 44, 330-338 (1973).

5. Williams, R., Crandall, R. S. \& Woitowicz, P. J. Phys. Rev. Lett. 37, 348-351 (1976).

6. Brown, J. C., Pusey, P. N., Goodwin, J. W. \& Ottewil, R. H. J. gen. Phys. A8, 664-682 (1975).

. Schaefer, D. W. \& Ackerson, B. J. Phys. Rev. Lett. 35, 1448-1451 (1975)

8. James, R. W. The Optical Principles of the Diffraction of X-Rays Ch. 5 (Cornell University Press, 1965)

9. James, R. W. The Optical Princples of the Diffraction of $X$-Rays $\mathrm{Ch} .8$ (Cornell University Press, 1965)

10. Alexander, S. \& McTague, J. Phys. Rev. Lett. 41, 702-705 (1978).

\section{A new phase in solid state arsenic}

\author{
A. N. Rodionov, R. I. Kalendarev, G. V. Tchikvaidze \\ \& J. A. Eiduss
}

Peter Stuchka Latvian State University, Riga, USSR

It has generally been considered that in the solid state, arsenic exists as four allotropic modifications-yellow (As(I)), amorphous (As(II)), orthorhombic (As(III)), and rhombohedral (As(IV)). Free energy content is lowest for the rhombohedral form. The other forms are metastable, the yellow form being the least stable, existing only at low temperatures and in the dark ${ }^{1}$. It is the yellow form which has been the least studied. Since the effect of temperature, as well as that of irradiation with light or $X$ rays, is to induce amorphisation in yellow arsenic, no $X$-ray structural data are yet available, and only indirect observations have been available. From these a cubic form has been tentatively assigned to yellow arsenic crystals. Spectroscopic data suggest molecular bonding in the crystal, with the $\mathbf{A s}_{4}$ molecule as the structural unit-a molecule previously observed in the vapour state ${ }^{2}$.

There is little information available on the thermodynamic properties of yellow arsenic. This is relevant to attempts to raise the thermal stability margin, thus opening the possibilities of practical application of the material for silverless photography. We have now designed and built a Calvet-type microcalorimeter for studying phase transitions in thin evaporated films. Using this device for differential thermal analysis (DTA) we have obtained DTA curves showing the existence of a previously unknown reversible (endothermic) phase transition within the metastable As(I) yellow form of arsenic which thus contains two states $\mathrm{As}^{1}(\mathrm{I})$ and $\mathrm{As}^{2}(\mathrm{I})$.

The transition curve for $\mathrm{As}(\mathrm{I})$ starts at $227 \mathrm{~K}$, reaching a maximum at $230 \mathrm{~K}$. Reversibility was tested by repeated heating-cooling cycles of the calorimeter, the heating being interrupted immediately after termination of endothermic phase transition.

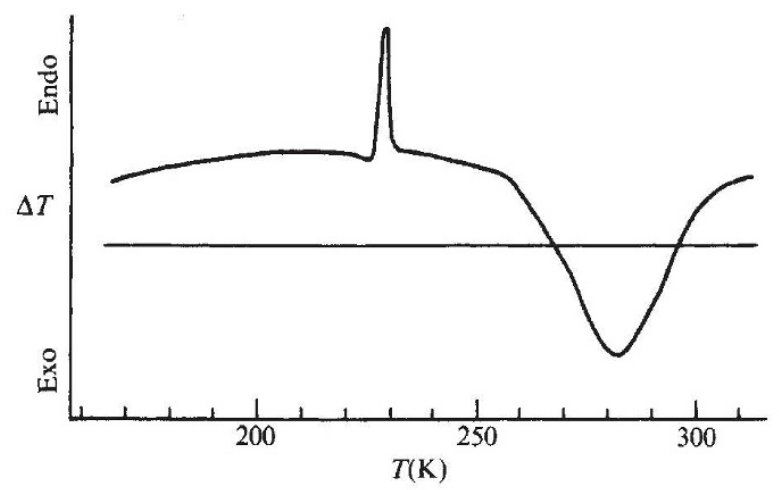

Fig. 1 DTA curve of thin evaporated film of yellow arsenic condensed at $205 \mathrm{~K}$.
Further heating causes the well known exothermic monotropic transition into amorphous arsenic As(II) with liberation of a considerable amount of heat. Both transitions are clearly visible on the thermographic curve shown on Fig. 1.

Direct structural measurements of yellow arsenic have so far proved unsuccessful, however, we can suggest certain estimates based on an analogy with phosphorus, the white form of which can be considered as an analogue of yellow arsenic. White phosphorus is also known to contain two allotropic forms, both based on $\mathrm{P}_{4}$ molecules as structural units, and differing only by additional rotational degree of freedom. Both forms of yellow arsenic- $\mathrm{As}^{1}(\mathrm{I})$ and $\mathrm{As}^{2}(\mathrm{I})$ - have also been found spectroscopically to contain $\mathrm{As}_{4}$ molecules which makes the analogy with phosphorus rather feasible.

Stability of the newly discovered yellow phase $\mathrm{As}^{2}(\mathrm{I})$ depends on the thickness of the yellow arsenic layer, the stability increasing with diminution of layer thickness. This may be due to an increase in surface energy with thinning of layer, leading to a rise in amorphisation temperature.

Received 4 June; accepted 27 June 1979.

1. Gmelin's Handbuch der anorganischen Chemie, 8th edn, 119 (Chemie Weinheim, 1952)

2. Capwell, R. \& Rosenblatt, G. J. molec. Spectr. 33, 525 (1970).

\section{Percolative conduction in microemulsion type systems}

\section{B. Lagourette, J. Peyrelasse, C. Boned \& M. Clausse*}

Université de PAU et des PAYS de l'ADOUR, Institut Universitaire de Recherche Scientifique, Département de Physique, Laboratoire de Thermodynamique, Avenue Philippon, F-64000 PAU France

In the presence of a suitable combination of surfactants (an alkaline metal soap and a medium chain length alcohol, for example), water and oil type organic liquids can form transparent compounds of low viscosity that have been labelled "microemulsions", with little or no mechanical agitation (spontaneous emulsification) (refs 1-3). Depending on the chemical nature of the surface active agents and the relative constituent proportions, water-in-oil (w/o) or oil-in-water (o/w) systems can be obtained. (Because of the similarity between tertiary solutions of inverted micelles and w/o microemulsions, the term 'inverted micellar solutions' has also been suggested ${ }^{4-7}$.) Microemulsions have been investigated by many scientists interested in liquid state and surface physicochemistry and by many technologists foreseeing numerous applications in industry $^{8,9}$. It has been suggested ${ }^{9-13}$ that conductivity and permittivity studies could provide, along with other techniques, valuable information about the structure and phase behaviour of microemulsions which are considered to consist of dispersions between a few tens and a few hundreds of angströms in diameter globules made up of an inner spherical core surrounded by a concentric shell of mixed surfactant and cosurfactant ${ }^{1-10}$. The experiments reported here show that the conductive behaviour of certain microemulsion systems can be accounted for using the percolation and effective-medium theories ${ }^{14-16}$ that have depicted transport properties and continuous metal-non-metal transitions in disordered materials with microscopic inhomogeneities associated with, for example, density, composition ${ }^{15}$ or bonding configuration ${ }^{17}$ fluctuations. This result, which could help in understanding the structural behaviour of microemulsions, is considered in connection with the postulated existence in liquid systems of equilibrium bicontinuous structures, a state described by Scriven' ${ }^{18}$ as 'related to ordinary liquids as porous media are to homogeneous solids'.

* To whom correspondence should be addressed. 